\title{
Avaliação da resistência à fratura por torção dos sistemas TF Adaptive e Hyflex CM
}

Evaluation of torsional fracture resistance of TF Adaptive and Hyflex CM systems

Evaluación de la resistencia a la fractura por torsión de los sistemas TF Adaptive e Hyflex CM

\author{
Rodrigo Zuccolotto Ferraz Caselli \\ ORCID: https://orcid.org/0000-0002-6089-6502 \\ Faculdade São Leopoldo Mandic, Brasil \\ E-mail: rodrigocaselli@hotmail.com \\ Cesar Augusto Perini Rosas \\ ORCID: https://orcid.org/0000-0002-2234-5531 \\ Universidade Estadual do Norte do Paraná, Brasil \\ E-mail: cesarperini66@hotmail.com \\ Ana Grasiela da Silva Limoeiro \\ ORCID: https://orcid.org/0000-0003-4633-720X \\ Faculdade de Ilhéus, Brasil \\ E-mail: grasielalimoeiro@gmail.com \\ Rina Andrea Pelegrine \\ ORCID: https://orcid.org/0000-0003-4175-2121 \\ Faculdade São Leopoldo Mandic, Brasil \\ E-mail: rinapelegrine@ terra.com.br \\ Carlos Eduardo Fontana \\ ORCID: https://orcid.org/0000-0002-2868-6839 \\ Pontifícia Universidade Católica de Campinas, Brasil \\ E-mail: ceffontana@hotmail.com \\ Emílio Henrique Rocha Gonçalves Ferreira \\ ORCID: https://orcid.org/0000-0002-6858-0772 \\ Faculdade São Leopoldo Mandic, Brasil \\ E-mail: emiliohenriquerocha@gmail.com \\ Rodrigo Casasanta França \\ ORCID: https://orcid.org/0000-0002-3248-5271 \\ Universidade de São Paulo, Brasil \\ E-mail: rodrigofranca@ alumni.usp.br \\ Marcelo dos Santos \\ ORCID: https://orcid.org/0000-0002-5364-4200 \\ Universidade de São Paulo, Brasil \\ E-mail: msantos@usp.br \\ Carlos Eduardo da Silveira Bueno \\ ORCID: https://orcid.org/0000-0002-2675-0884 \\ Faculdade São Leopoldo Mandic, Brasil \\ E-mail: carlosesbueno@terra.com.br
}

\begin{abstract}
Resumo
O presente estudo teve como objetivo avaliar a resistência à fratura por torção de dois sistemas de instrumentação baseados em diferentes cinemáticas de movimento: o movimento alternado do sistema TF Adaptive e o movimento rotatório do sistema Hyflex CM. Para isto foram utilizados 15 instrumentos TF Adaptive de 27 mm de comprimento, com as seguintes características: 20/.04, 25/.06, 35/.04 e 30 instrumentos Hyflex CM de 25 mm de comprimento, com as seguintes características: 25/.08, 20/.04, 25/.04, 20/.06, 30/.04 e 40/.04. Os instrumentos foram avaliados anteriormente ao seu uso e após dois, quatro, seis e oito usos em canais simulados em blocos de resina acrílica padronizados cuja curvatura do canal era de 35 graus e seu comprimento final de $18 \mathrm{~mm}$, sendo esse ponto alcançado ao final da instrumentação em ambos os sistemas empregados. A variável em questão foi a medida em graus até o momento da fratura do instrumento, obtida por meio de um Troptômetro modificado. Os valores registrados no momento da fratura, para cada instrumento, foram submetidos à análise estatística utilizando o teste de Wilcoxon Mann-Withney U Test para a comparação entre os dois sistemas de instrumentação. Os resultados mostraram que ambos os instrumentos não apresentaram diferenças quando avaliados sem nenhum uso, após dois usos, quatro usos, seis usos e por fim oito usos ( $\mathrm{p} \geq 0.05$ ). Concluiu-se que os sistemas TF Adaptive e Hyflex CM apresentaram similaridade quanto a resistência à fratura por torção.
\end{abstract}

Palavras-chave: Endodontia; Anomalia torcional; Resistência à fratura. 


\begin{abstract}
The present study had as objective to evaluate the resistance to torsion fracture of two instrumentation systems based on different motion cinematics: alternating movement of the system TF Adaptive and the rotary movement of the system HYFLEX CM. For this $15 \mathrm{TF}$ Adaptive instruments were used to $27 \mathrm{~mm}$ in length, with the following dimensions: 20/.04, 25/.06, 35/04 and 30 Hyflex CM instruments to $25 \mathrm{~mm}$ in length, with the following dimensions: $25 / .08,20 / .04,25 / 04,20 / .06,30 / .04$ and 40/.04. The instruments were evaluated prior to your use and after two, four, six and eight use in simulated acrylic resin canals. These canals were standardized and their curvature was 35 degrees and their final length $18 \mathrm{~mm}$, being this point reached at the end of the instrumentation in both systems. The variable in question was measured in degrees until the time of instrument fracture, obtained through a modified Troptômetro. The values registered at the time of fracture, for each instrument, were submitted to statistical analysis using the Wilcoxon test, Mann-Whitney U Test for comparison between the two systems of instrumentation ( $p \geq 0.05)$. The results showed that both instruments did not show differences when evaluated without any use, after two uses, four uses, six uses and finally eight uses $(\mathrm{p} \geq 0.05)$. It was concluded that TF Adaptive systems and Hyflex CM showed similarity as resistance to torsion fracture.
\end{abstract}

Keywords: Endodontics; Torsion abnormality; Fracture resistance.

\title{
Resumen
}

El presente estudio tuvo como objetivo evaluar la resistencia a la fractura por torsión de dos sistemas de instrumentación basados en diferentes cinemáticas de movimiento: el movimiento alterno del sistema TF Adaptive y el movimiento rotacional del sistema Hyflex CM. Para ello se utilizaron 15 instrumentos TF Adaptive de 27 mm de longitud, con las siguientes características: 20 / .04, 25 / .06, 35 / .04 y 30 instrumentos Hyflex CM de 25 mm de longitud, con las siguientes características: 25 /.08, $20 / .04,25 / .04,20 / .06,30 / .04$ y 40 / .04. Los instrumentos fueron evaluados antes de su uso y después de dos, cuatro, seis y ocho usos en canales simulados en bloques de resina acrílica estandarizada cuya curvatura del canal fue de 35 grados y su longitud final fue de $18 \mathrm{~mm}$, alcanzándose este punto al final de la instrumentación. en ambos sistemas empleados. La variable en cuestión fue la medición en grados hasta el momento de la fractura del instrumento, obtenida mediante un Troptómetro modificado. Los valores registrados en el momento de la fractura, para cada instrumento, se sometieron a análisis estadístico mediante la prueba U de Wilcoxon Mann-Withney para comparar los dos sistemas de instrumentación. Los resultados mostraron que ambos instrumentos no presentaron diferencias al ser evaluados sin ningún uso, luego de dos usos, cuatro usos, seis usos y finalmente ocho usos ( $\mathrm{p} \geq 0.05)$. Se concluyó que los sistemas TF Adaptive e Hyflex CM eran similares en términos de resistencia a la fractura por torsión.

Palabras clave: Endodoncia; Anomalía torsional; Resistencia a la fractura.

\section{Introdução}

As ligas de níquel-titânio representam um grande avanço na endodontia, particularmente no que diz respeito ao preparo de canais radiculares curvos. Tais ligas promoveram um aumento significativo na flexibilidade dos instrumentos devido ao seu baixo módulo de elasticidade, permitindo o desenvolvimento e a fabricação de novas formas geométricas em termos de secção reta transversal e conicidade. Esta propriedade evidencia um mínimo ou nenhum transporte do canal (Lopes \& Elias, 2001). Segundo Berutti et al., (2003), os instrumentos de NiTi, quando comparados aos instrumentos de aço inoxidável, são duas ou três vezes mais flexíveis e superiores em termos de deflexão angular, propriedades essas que favorecem a manutenção da forma do canal e a posição original do forame. Apesar de todos esses avanços nos instrumentos de $\mathrm{NiTi}$, o risco de fratura continua a ser um problema durante a terapia endodôntica e ela pode vir a ocorrer de duas formas principais: por fadiga torcional ou por fadiga flexural (cíclica). A fratura por torção ocorre quando a ponta do instrumento ou qualquer parte do mesmo prende-se às paredes do canal enquanto o eixo do instrumento continua a girar (Lopes et al., 2010). Já a fratura por flexão ocorre quando o instrumento gira repetidas vezes no interior de um canal curvo, gerando tensões alternadas de tração e compressão, as quais podem levar o instrumento à fratura (Moreira et al., 2002).

Nos últimos anos, tratamentos térmicos são aplicados à liga metálica a fím de garantir ao instrumento maior flexibilidade e resistência. Dois sistemas representantes dessa geração são as limas Hyflex CM (Coltene, Whaledent USA), que apresentam memória controlada, e as limas TF Adaptive (SybronEndo, Orange, EUA), que apresentam um tratamento térmico denominado R-phase. 
As limas Hyflex CM são produzidas utilizando um processo único no qual a fase cristalográfica transita de austenita para martensita em temperatura ambiente, apresentam menor quantidade de níquel contrastando com as limas NiTi tradicionais, tornando as limas extremamente flexíveis e resistentes a fratura (Testarelli et al., 2011). Em função da memória controlada, as limas tendem a seguir a anatomia do canal, e desta forma reduzem de forma significativa o risco de confecção de degraus, transporte ou perfuração do canal. Além disso, são limas capazes de recuperar o seu formato original e regenerar as estruturas de cristal e a resistência a fadiga após um processo normal de autoclave. No caso, da lima falhar na recuperação do seu formato após o tratamento com calor, estará plasticamente deformada e, portanto, terá o risco de fratura aumentado (Shen et al., 2013).

Em contrapartida, os instrumentos TF são fabricados com a liga de NiTi com estrutura cristalina de transição, denominada fase $\mathrm{R}$, obtida por um processo de resfriamento e aquecimento do fio metálico primitivo. Eles são fabricados pela torção de uma haste metálica com forma piramidal e secção reta transversal triangular. Segundo o fabricante, este processo de fabricação aumenta a dureza do material e melhora a resistência mecânica a fratura em torção e flexão (Lopes et al.,2010). Outra peculiaridade desse sistema é que, segundo o fabricante, deve ser utilizado em motor próprio, que realiza um movimento único, patenteado, a partir do qual, inicialmente, o instrumento é acionado no sentido horário, em rotação contínua, permitindo maior eficiência de corte, porém, havendo alguma resistência no trabalho do instrumento, a cinemática modifica-se, automaticamente, para movimentos no sentido horário e anti-horário, variando de $600^{\circ}-0^{\circ}$ até $370^{\circ}-50^{\circ}$. Com isso, o instrumento teria melhores condições de ultrapassar o ponto de dificuldade e, uma vez diminuído o stress sobre o instrumento, o movimento voltaria a ser de rotação contínua (Silva et al., 2017). Essa cinemática foi denominada Adaptive Motion.

Dentre os fatores que mais podem influenciar a fratura do instrumento endodôntico estão as condições anatômicas, como os raios e ângulos de curvatura do canal radicular, o comprimento do arco, o número de usos do instrumento, o torque utilizado e a experiência do operador (Haikel et. al., 1999).

Para avaliar a resistência à fratura por torção de um instrumento, utiliza-se de um ensaio mecânico de torção. $\mathrm{O}$ ensaio de torção consiste em aplicar uma força no corpo de prova para induzir um movimento de rotação em torno do seu centro de resistência. Para execução deste ensaio, fixa-se uma das extremidades do corpo de prova e aplica-se um movimento na extremidade oposta (Elias \& Lopes, 2007). Nesse presente estudo, foi utilizado um dispositivo modificado por França e Santos (2014) chamado Troptômetro.

Mediante essas constatações, torna-se pertinente a avaliação da resistência à fratura por torção dos instrumentos rotatórios com a finalidade de conceder ao profissional uma maior segurança em relação a seu uso. Portanto, o objetivo desse trabalho, foi avaliar a resistência à fratura por torção de dois sistemas de instrumentação baseados em diferentes cinemáticas de movimento: o movimento alternado do sistema TF Adaptive e o movimento rotatório do sistema Hyflex CM. A hipótese nula testada foi de que os instrumentos avaliados não apresentassem diferenças quanto à resistência à fratura por torção.

\section{Metodologia}

Para elaboração e execução da pesquisa foram utilizados os seguintes materiais que estão no Quadro 1. 
Quadro 1. Quadro de materiais e equipamentos.

\begin{tabular}{|c|c|c|}
\hline PRODUTO & FABRICANTE & ORIGEM \\
\hline ANÉIS DE COBRE & $\begin{array}{l}\text { COPPER BANDS (SEAM LESS), } \\
\text { INDÚSTRIA ODONTOLÓGICA } \\
\text { EVANG LTDA }\end{array}$ & SÃO PAULO (BRASIL) \\
\hline $\begin{array}{l}40 \text { CANAIS SIMULADOS } \\
\text { PADRONIZADOS EM RESINA } \\
\text { EPÓXICA }\end{array}$ & FUNAK & SÃO PAULO (BRASIL) \\
\hline $\begin{array}{l}\text { 05 CAIXAS DE LIMAS TF } \\
\text { ADAPTIVE }\end{array}$ & SYBRON ENDO & ORANGE (EUA) \\
\hline $\begin{array}{l}\text { 05 CAIXAS DE LIMAS HYFLEX } \\
\text { CM }\end{array}$ & COLTENE/WHALEDENT & OHIO(EUA) \\
\hline $\begin{array}{l}\text { 04 CAIXAS DE LIMAS MANUAIS } \\
\text { TIPO K 06, 08,10 e } 15\end{array}$ & MAILLEFER & BALLAIGUES (SUIÇA) \\
\hline GLICERINA & FARMAX & SÃO PAULO (BRASIL) \\
\hline GAZE & MB TESTIL CIRÚRGICA & GOIANIA (BRASIL) \\
\hline MORSA DE APREENSÄO & FERMEC & SÃO PAULO (BRASIL) \\
\hline MOTOR ELEMENTS & SYBRON ENDO & ORANGE (EUA) \\
\hline $\begin{array}{l}\text { TROPTÔMETRO MODIFICADO } \\
\text { SEGUNDO FRANÇA E SANTOS }\end{array}$ & SANTOS E FRANÇA & SÃO PAULO (BRASIL) \\
\hline SERINGA PLÁSTICA DE 10 ML & $\begin{array}{l}\text { PLASTIPAK-BECTON E } \\
\text { DICKINSON IND.CIR.LTDA }\end{array}$ & SÃO PAULO (BRASIL) \\
\hline AGULHA PARA IRRIGAÇÁO & INJEX 27 G 25X0,40 & SÃO PAULO (BRASIL) \\
\hline RÉGUA MILIMETRADA & DENTYSPLY & BALLAIGUES (SUIÇA) \\
\hline HIPOCLORITO DE SÓDIO 0,5\% & FÓRMULA E AÇÁO & SÃO PAULO (BRASIL) \\
\hline $\begin{array}{l}\text { 01 CANETA RETROPROJETOR } \\
\text { PONTA MÉDIA }\end{array}$ & FABER-CASTELL & SÃO PAULO (BRASIL) \\
\hline $\begin{array}{l}\text { 01 CÂNULA METÁLICA PARA } \\
\text { ASIPRAÇÁO 40:20 E 40:10 }\end{array}$ & $\begin{array}{l}\text { IBRAS-CBO IND.CIRÚRGICAS E } \\
\text { ÓPTICAS S.A. }\end{array}$ & SÃO PAULO (BRASIL) \\
\hline
\end{tabular}

Fonte: Autores.

\subsection{Delineamento experimental}

Esse trabalho laboratorial de caráter quantitativo foi baseado no trabalho de França e Santos (2014). O presente trabalho foi dispensado pelo Comitê de Ética e Pesquisa por tratar-se exclusivamente de pesquisa laboratorial, sem envolvimento de seres humanos ou materiais biológicos.

Para a avaliação da resistência à fratura por torção foram utilizados 15 instrumentos TF Adaptive de $27 \mathrm{~mm}$ de comprimento (Figura 1), com as seguintes características: 20/.04, 25/.06, 35/.04 e 30 instrumentos Hyflex CM de 25 mm de comprimento (Figura 2), com as seguintes características: 25/.08, 20/.04, 25/.04, 20/.06, 30/.04 e 40/.04.

Os instrumentos foram avaliados anteriormente ao seu uso e após dois, quatro, seis e oito usos em canais simulados em blocos de resina acrílica (Figura 3).

A variável em questão foi a medida em graus, até o momento da fratura do instrumento. O aparelho utilizado para se obter essa medida foi o Troptômetro modificado segundo França \& Santos (2014) (Figura 4). 
Research, Society and Development, v. 10, n. 14, e28101421604, 2021

(CC BY 4.0) | ISSN 2525-3409 | DOI: http://dx.doi.org/10.33448/rsd-v10i14.21604

Figura 1. Limas TF Adaptive.

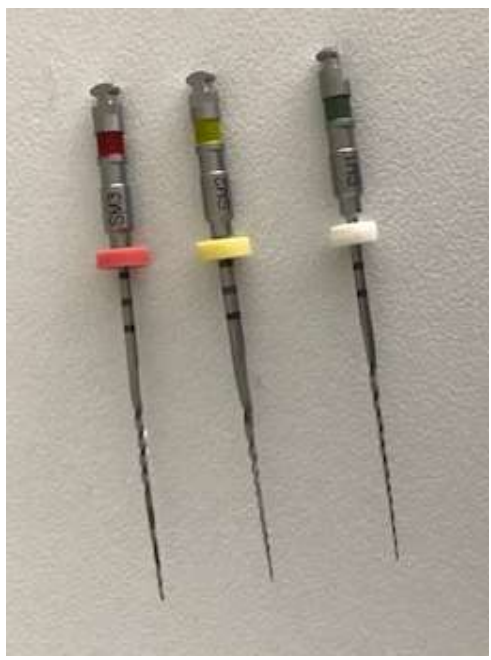

Fonte: Autores.

Figura 3. Blocos de resina acrílica

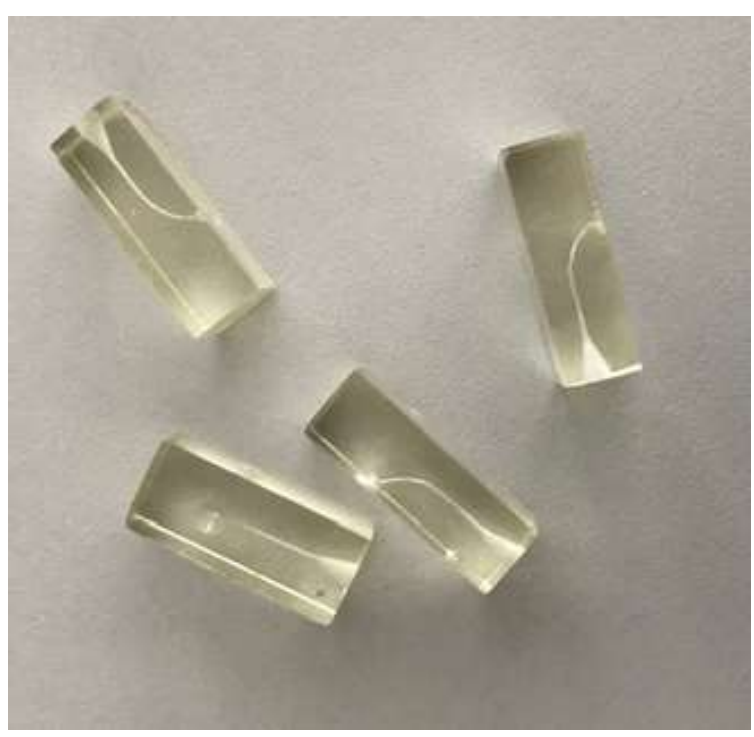

Fonte: Autores.
Figura 2. Limas Hyflex CM.

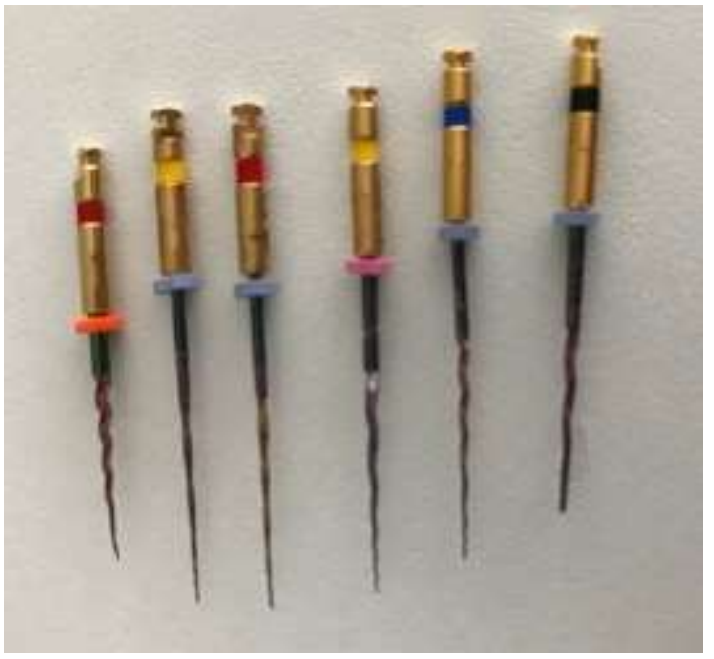

Fonte: Autores.

Figura 4. Troptômetro modificado

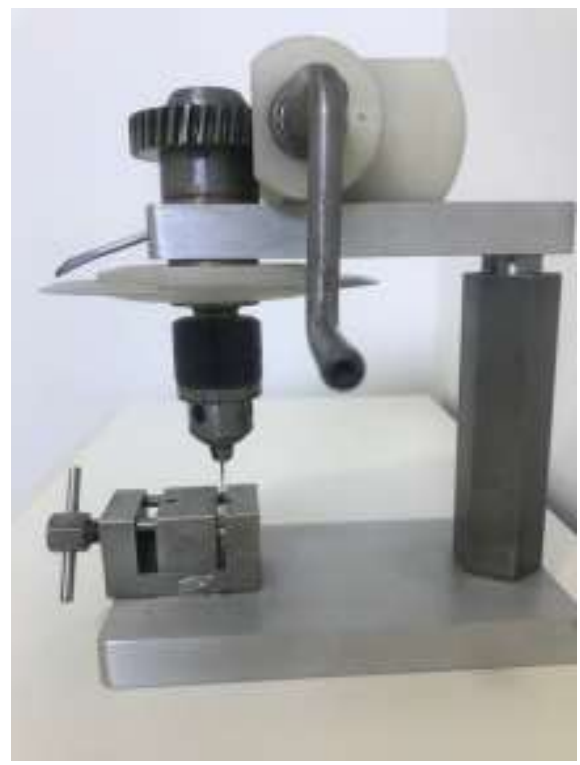

Fonte: Autores.

\subsection{Grupos experimentais}

Dez grupos experimentais foram formados, de acordo com o sistema de instrumentação empregado e o número de usos (Quadro 2). 
Quadro 2. Grupos experimentais com o tipo de instrumento e número de usos.

\begin{tabular}{|l|l|}
\hline GTFA 0 (SEM NENHUM USO) & $20 / .04,25 / .06,35 / .04$ \\
\hline GTFA 2 (APÓS 2 USOS) & $20 / .04,25 / .06,35 / .04$ \\
\hline GTFA 4 (APÓS 4 USOS) & $20 / .04,25 / .06,35 / .04$ \\
\hline GTFA 6 (APÓS 6 USOS) & $20 / .04,25 / .06,35 / .04$ \\
\hline GTFA 8 (APÓS 8 USOS) & $20 / .04,25 / .06,35 / .04$ \\
\hline GHF 0 (SEM NENHUM USO) & $25 / .08,20 / .04,25 / .04,20 / .06,30 / .04,40 / .04$ \\
\hline GHF 2 (APÓS 2 USOS) & $25 / .08,20 / .04,25 / .04,20 / .06,30 / .04,40 / .04$ \\
\hline GHF 4 (APÓS 4 USOS) & $25 / .08,20 / .04,25 / .04,20 / .06,30 / .04,40 / .04$ \\
\hline GHF 6 (APÓS 6 USOS) & $25 / .08,20 / .04,25 / .04,20 / .06,30 / .04,40 / .04$ \\
\hline GHF 8 (APÓS 8 USOS) & $25 / .08,20 / .04,25 / .04,20 / .06,30 / .04,40 / .04$ \\
\hline
\end{tabular}

Fonte: Autores.

Inicialmente, uma sequência completa de limas TF Adaptive e Hyflex CM, sem nenhum uso, foram submetidas ao teste de resistência à fratura por torção utilizando o troptômetro modificado segundo França e Santos (2014). Em seguida, uma segunda sequência de instrumentos foi utilizada para instrumentar dois canais simulados. Feito isso, uma terceira sequência foi usada para instrumentar quatro canais, seguida de uma quarta sequência para instrumentar seis canais e por fim, uma última sequência para preparar oito canais simulados. Ao final de cada grupo de instrumentação, todos os instrumentos foram submetidos ao teste de torção com a finalidade de se medir a resistência à fratura dos instrumentos. Todos os instrumentos testados dos respectivos fabricantes eram do mesmo lote.

\subsection{Instrumentação dos canais simulados}

Durante a instrumentação, foram utilizados blocos de resina epóxica padronizados cuja curvatura do canal simulado era de 35 graus e seu comprimento final de $18 \mathrm{~mm}$, sendo esse ponto alcançado ao final da instrumentação em ambos os sistemas empregados.

Esses blocos foram fixados em um torno para facilitar a imobilização durante a realização dos experimentos. A instrumentação foi iniciada com limas manuais 06, 08,10 e 15 para ambos os sistemas avaliados, até o comprimento total do bloco. Em seguida, foi realizada a instrumentação mecanizada com a sequência preconizada pelo fabricante. Para as limas TF Adaptive, utilizou-se a sequência 20/.04, 25/.06, 35/.04, com movimentos suaves de entrada e saída com pincelamento no retorno das limas, todas chegando ao comprimento de trabalho, determinado pelo tamanho total do bloco (18mm) e acionadas pelo motor Elements (SybronEndo) no movimento adaptive, com velocidade e torque já programados pelo fabricante. Para os blocos instrumentados com as limas Hyflex CM, após a instrumentação com limas manuais 06, 08,10 e 15, todas no comprimento de trabalho, foi feita a instrumentação mecanizada com a sequência proposta pelo fabricante (25/.08, 20/.04, 25/.04, 20/.06, 30/.04 e 40/.04). Apenas a lima 25/.08 não foi levada até o comprimento de trabalho e sim apenas até o início da curvatura. Também foram utilizadas no motor Elements, com movimentos suaves de entrada e saída com pincelamento no retorno da lima, porém no movimento de rotação contínua com velocidade de 500 RPM e torque 2,5N.cm. Entre cada lima, tanto no protocolo da TF Adaptive quanto no da Hyflex CM, o canal simulado foi irrigado com hipoclorito de sódio a 0,5\% e aspirado com cânulas metálicas para aspiração, totalizando $10 \mathrm{ml}$ para cada bloco instrumentado. Foi utilizado também glicerina entre cada lima para lubrificação do canal e gaze umedecida com hipoclorito de sódio na mesma concentração para remoção de raspas de resina aderida as limas.

\subsection{Testes de torção}

Após cada etapa pré-determinada de duas, quatro, seis e oito utilizações, os instrumentos respectivos foram levados ao teste de torção para avaliação de sua resistência em graus à fratura, utilizando o Troptômetro modificado, segundo França e 
Santos (1994).

Cada instrumento recebeu uma marca com uma caneta de tinta permanente na altura do seu diâmetro $\mathrm{D}^{3}$ (3 mm da ponta de cada instrumento), medida realizada com uma régua milimetrada. A seguir foi posicionado o instrumento, um por vez, no mandril de modo a coincidir a marca com o plano determinado pelo limite superior das garras de apreensão.

Estando o instrumento convenientemente posicionado, a escala do aparelho foi calibrada em zero grau. Foi girado, então, o parafuso movimentador das garras de apreensão até se obter pressão suficiente para que as lâminas de cobre ficassem estampadas no formato dos instrumentos. Esse procedimento teve por finalidade impedir o movimento rotacional livre da extremidade ativa, bem como reduzir a possibilidade de indução de zonas de fragilidade derivadas de deformações provocadas pela compressão das partes. A cada ensaio, foram substituídas as lâminas de cobre.

Ao se executar o teste, a manivela acionadora das engrenagens foi vagarosamente movimentada fazendo com que o mandril girasse no sentido horário. A manivela foi acionada de modo a permitir a progressão de aproximadamente um grau a cada segundo, com a finalidade de se parar no momento da fratura do instrumento. A seguir, lia-se na escala do aparelho a quantidade de graus percorridos desde 0 grau até o momento da fratura. Os valores registrados neste momento, para cada instrumento, foram anotados e procedeu-se a análise estatística pertinente.

\subsection{Análise estatística}

Nesse estudo foi utilizado o teste deWilcoxon Mann-Withney U Test para a comparação entre os dois sistemas de instrumentação. O nível de significância assumido para decidir sobre todos os testes foi de 5\%, ou seja, de $95 \%$ de confiança.

\section{Resultados}

Os resultados mostraram que não houve diferenças estatisticamente significantes com relação aos sistemas TF Adaptive e Hyflex CM quando esses foram avaliados em diferentes números de uso ( $\mathrm{p} \geq 0.05$ ) (Quadro 3).

Quadro 3. Análise comparativa entre os sistemas TF Adaptive e Hyflex CM com relação à resistência a fratura por torção nos diferentes tempos de uso.

\begin{tabular}{|c|l|l|l|}
\hline TEMPO DE USO & \multicolumn{1}{|c|}{ TFADAPTIVE } & \multicolumn{1}{|c|}{ HYFLEX CM } & \\
\hline 0 & $623.33+/-82,81$ & $643.83+/-129,68$ & $\mathrm{P}=0,89$ \\
\hline 2 & $556.33+/-69,34$ & $595.00+/-100,73$ & $\mathrm{P}=0,60$ \\
\hline 4 & $535.00+/-44,31$ & $573.50+/-73,59$ & $\mathrm{P}=0,30$ \\
\hline 6 & $520.33+/-53,89$ & $562.33+/-121,76$ & $\mathrm{P}=1,00$ \\
\hline 8 & $477.67+/-27,43$ & $504.50+/-60,36$ & $\mathrm{P}=0,25$ \\
\hline
\end{tabular}

Fonte: Autores.

O quadro a seguir mostra a variação em graus, para cada instrumento de cada sistema, até que ocorresse a fratura (Quadro 4). 
Quadro 4. Variação em graus de cada sistema.

\begin{tabular}{|l|l|l|l|l|l|l|}
\hline Sistemas & $\begin{array}{l}\text { Num. do } \\
\text { instr. }\end{array}$ & $\begin{array}{l}\text { Sem } \\
\text { instrument. }\end{array}$ & $\begin{array}{l}\text { Duas } \\
\text { instrument. }\end{array}$ & $\begin{array}{l}\text { Quatro } \\
\text { instrument. }\end{array}$ & $\begin{array}{l}\text { Seis } \\
\text { instrument. }\end{array}$ & $\begin{array}{l}\text { Oito } \\
\text { instrument. }\end{array}$ \\
\hline Hyflex CM & 25.08 & 838 graus & 759 graus & 655 graus & 680 graus & 574 graus \\
& 20.04 & 698 graus & 601 graus & 630 graus & 658 graus & 435 graus \\
& 25.04 & 518 graus & 532 graus & 492 graus & 455 graus & 481 graus \\
& 20.06 & 652 graus & 578 graus & 612 graus & 681 graus & 579 graus \\
& 30.04 & 676 graus & 638 graus & 574 graus & 462 graus & 503 graus \\
& 40.04 & 481 graus & 462 graus & 478 graus & 438 graus & 455 graus \\
\hline TF Adapt . & 20.04 & 652 graus & 538 graus & 484 graus & 502 graus & 458 graus \\
& 25.06 & 530 graus & 498 graus & 557 graus & 478 graus & 509 graus \\
& 35.04 & 688 graus & 633 graus & 564 graus & 581 graus & 466 graus \\
\hline
\end{tabular}

Fonte: Autores.

\section{Discussão}

A hipótese nula foi aceita, pois os instrumentos avaliados não apresentaram diferenças quanto a resistência à fratura por torção.

A literatura é extensa ao relatar que a liga de NiTi é mais flexível que a até então utilizada de aço inoxidável, propriedade fundamental para um instrumento endodôntico. Outras propriedades interessantes que a liga de NiTi possui deixando-a mais vantajosa são: superelasticidade, efeito memória de forma e biocompatibilidade (Pruett et al.,1997; Thompson, 2000; Melo et al., 2002; Lopes et al., 2010; Gutmann e Gao, (2012).

O uso das limas de NiTi mecanizadas apresentava um inconveniente: a resistência mecânica, uma vez que quando submetida a repetidos esforços, associada a substâncias químicas auxiliares, ciclos de esterilizações e habilidade do operador tornam-se susceptíveis a fratura, que pode ocorrer por fadiga torcional, cíclica ou a sua combinação (Lopes et al.,2010). Vale ressaltar que a fratura dos instrumentos de NiTi geralmente ocorre sem que o operador previamente perceba, pois, o instrumento raramente apresenta qualquer tipo de deformação ou defeito visível a olho nú (Pruett et al.,1997).

As cinemáticas utilizadas na instrumentação dos canais radiculares frequentemente acabam gerando uma pressão na ponta do instrumento a cada vez que ele é introduzido no canal, milímetro a milímetro. Blum et al. (1999) afirmaram que os instrumentos endodônticos sofrem maior "stress" durante o movimento de entrada do instrumento no interior dos canais radiculares, e não durante sua remoção. Assim, se esta pressão apical for excessiva, o risco deste instrumento travar junto às paredes dentinárias é grande, o que poderia determinar a fratura do instrumento neste ponto. E, com a proposta de diminuir o stress no instrumento durante o preparo, foi lançado o movimento adaptive (alternado), uma vez que, havendo interferência no canal o movimento passa, automaticamente, de rotação contínua para reciprocante até que a dificuldade seja ultrapassada (Silva et al., 2017).

Tratamentos térmicos, aprimoramento no desenho, diferentes secções transversais e formas de confecção dos instrumentos, sejam estes por usinagem ou torção, além de cinemáticas de movimento diferentes, foram estudadas e desenvolvidas a fim de proporcionar ao cirurgião dentista maior segurança em relação à fratura do instrumento durante a terapia endodôntica (Lopes \& Elias, 2001). A proposta desse estudo foi avaliar a resistência à fratura por torção de dois sistemas de instrumentação baseados em diferentes cinemáticas de movimento: o movimento alternado por meio do sistema $\mathrm{TF}$ Adaptive e o movimento rotatório por meio do sistema Hyflex CM. Características peculiares destes instrumentos os fizeram escolhidos para este estudo, como por exemplo, o processo especial termomecânico que controla a memória do material empregado na lima Hyflex CM, fazendo com que esses instrumentos se tornem extremamente flexíveis e altamente resistentes (Shen et al., 2011) e o processo de torção em seu fio fase R empregado na lima TF Adaptive juntamente com seu movimento alternado, que vem com a proposta de ser mais flexível que as concorrentes e conferir maior resistência ao instrumento. Os 
resultados desse trabalho mostraram que o movimento alternado não influenciou na resistência à fratura por torção e, estatisticamente, ambos os instrumentos não apresentaram diferenças quando avaliados sem nenhum uso, após dois usos, quatro usos, seis usos e por fim oito usos ( $\mathrm{p} \geq 0.05)$.

Estudos de Castelló-Escrivá et al., 2012, Pedullá et al., 2013, Higuera et al., 2015 mostraram melhores resultados mecânicos e de preparo no sistema TF Adaptive em comparação a sistemas reciprocantes, como Reciproc e WaveOne, e sistemas rotatórios como Mtwo. Esses resultados são promissores em relação a esta cinemática combinada, tornando o preparo mais seguro para casos de curvaturas ou canais atrésicos. Contudo, é necessário avaliar se os resultados deste sistema se explicam somente pela sua cinemática particular, ou também por suas características de secção transversal, de conicidade, de tamanho de ponta dos instrumentos ou de tratamento térmico.

Elnaghy e Elsaka (2018) compararam a resistência à fadiga cíclica dos instrumentos XP-endo Shaper (FKG, Dentaire, La Chaux-de-Fonds, Suíça) com TRUShape (Dentsply, Tulsa Dental Specialties, Tulsa, USA), HyFlex CM (Coltene, Cuyahoga Falls, USA), Vortex Blue (Dentsply, Tulsa Dental Specialties, USA) e instrumentos rotativos de níquel-titânio iRace ( FKG, Dentaire La chaux-de-Fonds, Suiça) à temperatura corporal. Os instrumentos possuíam o mesmo tamanho (\#30), com conicidades diferentes e foram testados em canais artificiais com ângulo curvatura de $60^{\circ}$ e raio de $3 \mathrm{~mm}$. Como resultado, os instrumentos XPS tiveram um maior número de ciclos para a fadiga comparados aos demais testados.

Wycoff e Berzins (2012) então comparou a Twisted File com instrumentos endodônticos com secção transversal semelhante, no caso EndoSequence e Vortex. Concluiu que as novas técnicas utilizadas na fabricação das Twisted File não as tornaram mais resistentes à torção. Neste estudo, também foi utilizado instrumentos com a mesma secção transversal, e o resultado foi que ambos os instrumentos apresentaram similaridade quanto a resistência à fratura por torção.

O instrumento W-File (TDK, China) possui características semelhantes ao WaveOne Gold, como a secção transversal em paralelogramo, além disso, os tamanhos de ponta e conicidade. Estes instrumentos ainda passam por um tratamento térmico que induz uma camada de óxido de titânio na superfície do instrumento, fazendo com que a cor que fique dourada. (Calefi et al., 2020).

Prados-Privado et al., (2019) avaliaram as propriedades de flexão e torção de quatro instrumentos endodônticos de níquel-titânio. A pesquisa comparou instrumentos ponta ISO 25 WaveOne, WaveOne Gold, Reciproc 25 e Reciproc Blue. Modelos tridimensionais foram criados usando software. Os maiores níveis de estresse foram registrados para WaveOne e Reciproc. Os resultados numéricos do teste de flexão mostraram que o WaveOne Gold é 86\% mais flexível que o WaveOne com uma deflexão de três milímetros. O Reciproc Blue foi 42,31\% mais flexível que o Reciproc com uma deflexão de 3 mm. O instrumento WaveOne resistiu ao maior estresse sob o teste de torção, seguido pelo Reciproc e Reciproc Blue.

Para Elsaka et al., (2017) o design triangular da secção transversal do Twisted File Adaptive pode resultar em uma alta concentração de tensão, enquanto que o design em forma de $\mathrm{S}$ da Reciproc produz um diâmetro de núcleo interno maior. A área de secção transversal da Reciproc (aproximadamente $129.136 \mu \mathrm{m}^{2}$ ) é maior do que a área da Twisted File Adaptive (aproximadamente $24.491 \mu \mathrm{m}^{2}$ ), o que poderia explicar sua maior resistência à torção.

Já em relação ao tratamento térmico dos instrumentos, Ha et al., (2013) estudou se a Fase R dos instrumentos TF Adaptive melhora a resistência à torção e a fadiga cíclica. Concluíram que os instrumentos tratados termicamente apresentaram mais resistência à fadiga cíclica, sem redução da força de torção. O mesmo concluiu Shen et al., (2015), onde em seu estudo, a Fase $\mathrm{R}$ aumentou apenas a resistência à fadiga cíclica, entretanto no teste de fadiga torcional os resultados foram semelhantes.

Braga et al., (2014), compararam os instrumentos com tratamento térmico M-Wire, CM e NiTi convencionais. As limas com tratamento térmico CM foram as que apresentaram maior resistência a fadiga, seguidas da M-Wire e NiTi convencionais. No presente estudo, instrumentos com tratamento térmico $\mathrm{CM}$ e instrumentos Fase $\mathrm{R}$ apresentaram similaridade em relação a resistência à fratura torcional. 
Dentre os fatores que podem influenciar a fratura do instrumento endodôntico estão às condições anatômicas, como os raios e ângulos de curvatura do canal radicular, o número de usos do instrumento, o torque utilizado, diâmetro e conicidade do instrumento e a experiência do operador (Haikel et. al.,1999). Por isso, no presente trabalho justifica-se a escolha em instrumentar um canal simulado com curvatura de 35 graus, o uso dos instrumentos em até oito vezes e instrumentos com diâmetros e conicidades variadas. Os resultados mostraram uma diminuição na resistência à fratura por torção dos instrumentos à medida que aumentamos o seu número de usos. Tongbaiyai e Torabinejad (1999) afirmaram que as falhas nos instrumentos ocorrem principalmente devido ao uso incorreto destes instrumentos e não devido à frequência de uso, sugerindo que instrumentos de NiTi podem ser usados em aproximadamente dez casos antes de serem descartados.

Wolcott e Himel (1997) concluíram que o aumento do diâmetro e da conicidade tornam o instrumento mais resistente à fratura por torção. Svec e Powers (1999) afirmaram que a resistência à torção está diretamente relacionada com o calibre do instrumento. Segundo os autores, as limas de maior calibre apresentam uma maior resistência à torção. Shimabuko (2000), Maia Filho (2003) e Guilford et al., (2005) também concluíram que os instrumentos rotatórios de maior conicidade tendem a apresentar maior resistência à torção quando comparados aos de menor conicidade. Para Matheus et al., (2008), quanto maior núcleo metálico tiver o instrumento, maior carga será necessária para que ocorra a fratura. Foi observado neste estudo que as lima Hyflex CM 25.08 e TF Adaptive 35.04 foram as que apresentaram uma maior resistência à fratura por torção, porém a lima Hyflex CM 40.04 apresentou um resultado inferior se comparada aos instrumentos Hyflex CM 25.08 e TF Adaptive 35.04. Segundo trabalho de Costa e Santos (2000) o aumento da massa metálica torna o instrumento mais rígido, o que leva uma diminuição da resistência à fratura por torção, compensado pelo benefício de aumentar a eficiência de corte do instrumento. Já para Schrader e Peters (2005), intercalar instrumentos de diferentes conicidades mostrou ser mais efetivo na prevenção de fratura por fadiga torcional.

Um fato interessante em relação à resistência dos instrumentos TF Adaptive à fratura por torção foi observado durante o presente estudo. Alguns instrumentos deste sistema tiveram suas espirais distorcidas, assim como uma grande deformação de sua parte ativa junto ao ponto de imobilização. Somente instrumentos deste sistema comportaram-se desta maneira. Assim, pode-se supor que um instrumento que é elaborado com fio que apresenta a fase $\mathrm{R}$ tende a ser mais flexível, permitindo uma maior quantidade de deformação elástica e plástica quando submetido a um torque similar, em comparação a um instrumento produzido por fio de NiTi convencional. Este fato é corroborado pelos resultados de outros autores (Gambarini et al., 2008; Larsen et al., 2009; Kim et al., 2010).

O treinamento do profissional pode interferir diretamente no controle da instrumentação automatizada, refletindo na incidência de complicações e acidentes, principalmente em canais curvos. Sattapan et al., (2000) relataram que as deformações observadas nos instrumentos rotatórios de NiTi estão associadas muito mais à maneira de como as limas são utilizadas do que especificamente ao número de usos durante a instrumentação de canais radiculares. Inclusive, a compreensão dos fatores que levam a fratura de instrumentos automatizados constituiu condição importantíssima para a prevenção desse tipo de imprevisto, já que direciona o clinico a desenvolver precaução e zelo com as técnicas de preparo do canal de forma embasada e consciente.

Tornam-se então importantes mais estudos para avaliar a resistência à fratura dos instrumentos endodônticos, a fim de oferecer ao operador maior segurança em relação a seu uso.

\section{Conclusão}

De acordo com os resultados obtidos no presente estudo, pode-se concluir que os sistemas TF Adaptive e Hyflex CM apresentaram similaridade quanto a resistência à fratura por torção. Esse trabalho foi uma pequena contribuição para a ciência, visto que existe uma infinidade de sistemas mecanizados no mercado. Seria oportuno novos trabalhos comparando outros sistemas mais modernos. 
Research, Society and Development, v. 10, n. 14, e28101421604, 2021

\section{Referências}

Berutti, E., Chiandussi, G., Gaviglio, I. \& Ibba A. (2003). Comparative analyses of torsion and bending stresses in two mathematical models of nickel titanium rotary instruments: ProTaper versus ProFile. Journal of endodontics, 29(1), 15-20.

Blum, J. Y., Cohen, A., Machtou, P. \& Micallef, J. P. (1999). Analysis of forces developed during mechanical preparation of extracted teeth using Profile NiTi rotary instruments. International endodontics journal, 32(1), 24-31.

Braga, L. C., Faria Silva, A. C., Buono, V. T., \& de Azevedo Bahia, M. G. (2014). Impact of heat treatments on the fatigue resistance of different rotary nickel-titanium instruments. Journal of endodontics, 40(9), 1494-1497.

Calefi, P. H. S., Osaki, R. B., Dal Evedove, N. F., Cruz, V. M., de Andrade, F. B. \& Alcalde, M. P. (2020). Cyclic and torcional fatigue resistance of W File and X1 Blue file reciprocating instruments. Dental Press Endodontics. 10(2), 60-66.

Castelló-Escrivá, R., Alegre-Domingo, T., Faus-Matoses, V., Román-Richon, S., \& Faus-Llácer, V. J. (2012). In vitro comparison of cyclic fatigue resistance of ProTaper, WaveOne, and Twisted Files. Journal of endodontics, 38(11), 1521-1524.

Costa, C. \& Santos, M. (2000). Resistência à torção de dois instrumentos endodônticos rotatórios de níquel-titânio. Pesq Odont Bras, 14(2), 165-168.

Elias, C. N. \& Lopes, H. P. (2007). Materiais dentários. Ensaios mecânicos. Livraria Santos, p-180.

Elnaghy, A., \& Elsaka, S. (2018). Cyclic fatigue resistance of XP-endo Shaper compared with different nickel-titanium alloy instruments. Clinical oral investigations, 22(3), 1433-1437.

Elsaka, S. E., Elnaghy, A. M., \& Badr, A. E. (2017). Torsional and bending resistance of WaveOne Gold, Reciproc and Twisted File Adaptive instruments. International endodontic journal, 50(11), 1077-1083.

França, R. C. \& Santos, M. (2014). Resistência à torção de instrumentos rotatórios de uso repetitivo: K3 Endo x ProTaper. Anuário ABO de artigos científicos, $118-124$.

Gambarini, G., Grande, N. M., Plotino, G., Somma, F., Garala, M., De Luca, M., \& Testarelli, L. (2008). Fatigue resistance of engine-driven rotary nickeltitanium instruments produced by new manufacturing methods. Journal of endodontics, 34(8), 1003-1005.

Guilford, W. L., Lemons, J. E., \& Eleazer, P. D. (2005). A comparison of torque required to fracture rotary files with tips bound in simulated curved canal. Journal of endodontics, 31(6), 468-470.

Gutmann, J. L., \& Gao, Y. (2012). Alteration in the inherent metallic and surface properties of nickel-titanium root canal instruments to enhance performance, durability and safety: a focused review. International endodontic journal, 45(2), 113-128.

Ha, J. H., Kim, S. K., Cohenca, N., \& Kim, H. C. (2013). Effect of R-phase heat treatment on torsional resistance and cyclic fatigue fracture. Journal of endodontics, 39(3), 389-393.

Haïkel, Y., Serfaty, R., Bateman, G., Senger, B., \& Allemann, C. (1999). Dynamic and cyclic fatigue of engine-driven rotary nickel-titanium endodontic instruments. Journal of endodontics, 25(6), 434-440.

Higuera, O., Plotino, G., Tocci, L., Carrillo, G., Gambarini, G., \& Jaramillo, D. E. (2015). Cyclic fatigue resistance of 3 different nickel-titanium reciprocating instruments in artificial canals. Journal of endodontics, 41(6), 913-915.

Kim, H. C., Yum, J., Hur, B., \& Cheung, G. S. (2010). Cyclic fatigue and fracture characteristics of ground and twisted nickel-titanium rotary files. Journal of endodontics, 36(1), 147-152.

Larsen, C. M., Watanabe, I., Glickman, G. N., \& He, J. (2009). Cyclic fatigue analysis of a new generation of nickel titanium rotary instruments. Journal of endodontics, 35(3), 401-403.

Lopes, H. P. \& Elias, C. N. (2001). Fratura dos instrumentos endodônticos de NiTi acionados a motor. Fundamentos teóricos e práticos. Revista Paulista Odontolologia, 58, 207-209.

Lopes, H. P., Elias, C. N. \& Siqueira-Junior, J. F. (2010). Instrumentos endodônticos. Endodontia, Biologia e Técnica. (3a ed.), Guanabara Koogan, 305-413.

Lopes, H. P., Elias, C. N., Siqueira-Junior, J. F. \& Batista, M. M. D. (2010). Fratura de instrumentos endodônticos: Fundamentos teóricos e práticos. Endodontia, Biologia e Técnica. (3a ed.), Guanabara Koogan, 481-505.

Maia-Filho, E. M. (2003). Avaliação comparativa da resistência à fratura de instrumentos de níquel-titânio acionados a motor submetidos a ensaios de torção [tese doutorado]. Araraquara, Faculdade de Odontologia da UNESP.

Matheus, T. C. U., Albuquerque, D. S. \& Lopes, H. P. (2008). K3 Endo x ProFile, avaliação da resistência à fratura empregando ensaio mecânico de torção. http://www.funak.com.br/Aval_resist.asp>.

Chaves Craveiro de Melo, M., Guiomar de Azevedo Bahia, M., \& Lopes Buono, V. T. (2002). Fatigue resistance of engine-driven rotary nickel-titanium endodontic instruments. Journal of endodontics, 28(11), 765-769.

Moreira, E. J. L., Lopes, H. P., Elias, C. N. \& Fidel, R. A. S. (2002). Fratura por flexão em rotação de instrumentos endodônticos de NiTi. Revista Brasileira Odontologia, 59(6), 412-414. 
Research, Society and Development, v. 10, n. 14, e28101421604, 2021 (CC BY 4.0) | ISSN 2525-3409 | DOI: http://dx.doi.org/10.33448/rsd-v10i14.21604

Pedullà, E., Grande, N. M., Plotino, G., Gambarini, G., \& Rapisarda, E. (2013). Influence of continuous or reciprocating motion on cyclic fatigue resistance of 4 different nickel-titanium rotary instruments. Journal of endodontics, 39(2), 258-261.

Prados-Privado, M., Rojo, R., Ivorra, C., \& Prados-Frutos, J. C. (2019). Finite element analysis comparing WaveOne, WaveOne Gold, Reciproc and Reciproc Blue responses with bending and torsion tests. Journal of the mechanical behavior of biomedical materials, 90, 165-172.

Pruett, J. P., Clement, D. J., \& Carnes, D. L., Jr (1997). Cyclic fatigue testing of nickel-titanium endodontic instruments. Journal of endodontics, 23(2), 77-85.

Sattapan, B., Nervo, G. J., Palamara, J. E., \& Messer, H. H. (2000). Defects in rotary nickel-titanium files after clinical use. Journal of endodontics, 26(3), $161-165$.

Schrader, C., \& Peters, O. A. (2005). Analysis of torque and force with differently tapered rotary endodontic instruments in vitro. Journal of endodontics, 31(2), 120-123.

Shen, Y., Qian, W., Abtin, H., Gao, Y. \& Haapasalo, M. (2011). Fatigue testing of controlled memory wire nickel-titanium rotary instruments. Journal of endodontics, 37 (7), 997-1001.

Shen, Y., Zhou, H. M., Zheng, Y. F., Peng, B. \& Haapasalo, M. (2013). Current challenges and concepts of the thermomechanical treatment of nickel-titanium instruments, Journal of endodontics, 39(2), 163-172.

Shen, Y., Riyahi, A. M., Campbell, L., Zhou, H., Du, T., Wang, Z., Qian, W., \& Haapasalo, M. (2015). Effect of a combination of torsional and cyclic fatigue preloading on the fracture behavior of K3 and K3XF instruments. Journal of endodontics, 41(4), 526-530.

Shimabuko, D. M. (2000). Avaliação da resistência a torção de limas endodônticas rotatórias de níquel-titânio, em função da sua conicidade e sistema de fixação da ponta [tese doutorado]. São Paulo, Faculdade de Odontologia da USP.

Silva, E., De-Deus, G., Souza, E., Versiani, M. \& Zuolo, M. (2017). O movimento reciprocante na endodontia. São Paulo, Santos Publicações, 75-104.

Svec, T. A. \& Powers, J. M. (1999). Effects of simulated clinical conditions on nickel-titanium rotary files. Journal of endodontics, 25(11), 759-60.

Testarelli, L., Plotino, G., Al-Sudani, D., Vincenzi, V., Giansiracusa, A., Grande, N. M. \& Gambarini, G. (2011). Bending properties of a nickel-titanium alloy with a lower percent by weight of nickel. Journal of endodontics, 37(9), 1293-5.

Thompson S. A. (2000). An overview of nickel-titanium alloys used in dentistry. International endodontic journal, 33(4), 297-310.

Tongbaiyai, C. \& Torabinejad, M. (1999). The durability of .04 taper rotary NiTi files after simulated clinical usage [abstract OR 42]. Journal of endodontics, 25(4), 292.

Wolcott, J. \& Himel, V. T. (1997). Torsional properties of nickel-titanium versus stainless steel endodontic files. Journal of endodontics, 23, $217-220$.

Wycoff, R. C. \& Berzins, D. W. (2012). An in vitro comparison of torsional tension properties of three different rotary nickel-titanium files with a similar cross-sectional desing. Journal of endodontics, 38, 1118-20. 